\title{
Utilisation and predictors of use of exposure therapy in the treatment of anxiety, OCD and PTSD in an Australian sample: a preliminary investigation
}

\author{
Karen Moses ${ }^{1 *}$, Craig Gonsalvez ${ }^{1}$ and Tanya Meade ${ }^{1,2}$
}

\begin{abstract}
Background: Cognitive-behavior therapy (CBT) is known to be an effective treatment for the anxiety and related disorders, with exposure therapy being a key component of this treatment package. However, research on the use of exposure therapy in clinical practice has presented mixed results, potentially due to differences in samples and training programs across countries. The present study aimed to extend upon existing research by examining the use and predictors of use of exposure therapy in a sample of psychologists working in clinical practice in Australia who treat clients with an anxiety disorder, obsessive compulsive disorder (OCD) or post-traumatic stress disorder (PTSD).
\end{abstract}

Methods: One hundred registered psychologists $\left(M_{\text {age }}=40.60 ; S D=10.78\right.$; range 23 to 71 years; 84\% female) participated in an online study investigating their clinical practices.

Results: Results suggested that while the general use of exposure therapy is high, the use of disorder specific techniques was considerably lower, particularly for anxiety disorders and PTSD but not OCD. Psychology registration status and level of training were positively associated with use of exposure therapy as was the experience in treating anxiety disorders.

Conclusions: These findings suggest that further or ongoing professional training may be required to optimize the use of disorder specific techniques.

Keywords: Anxiety, Exposure therapy, CBT, Clinical practice, Treatment

\section{Introduction}

Anxiety, obsessive compulsive disorder (OCD) and posttraumatic stress disorder (PTSD) are among the most frequently experienced mental health problems $[1,2]$, tend to be long-lasting [3-6] and are associated with significant functional impairments across the lifespan [7-11]. Fortunately, research demonstrates that effective

\footnotetext{
${ }^{*}$ Correspondence: K.Moses@westernsydney.edu.au

1 School of Psychology, Western Sydney University, Locked Bag 1797,

Penrith, NSW 2751, Australia

Full list of author information is available at the end of the article
}

treatments for the anxiety disorders are available $[12,13]$, particularly when preceded by an evidence based assessment [14]. CBT is considered the first line intervention for the anxiety and related disorders, both nationally [15, 16] and internationally [17]. In Australia, significant public funding is available for the use of CBT when treating the anxiety and related disorders [15] and CBT, in particular exposure therapy, is one of the key evidence-based psychological interventions taught in professional training programs $[15,18-20]$. Whilst these disorders are no longer clustered together in the DSM 5 [21] under the umbrella term of 'anxiety disorders', given similarities in 
treatment approach, research has historically and continues to consider these disorders collectively [12, 18, 22-24]. For the purposes of the current research, this convention has been adopted.

Whilst the present research focuses primarily on the use of exposure therapy for the anxiety and related disorders, research to date largely considers and evaluates the use of exposure therapy as a key component of treatment in combination with cognitive interventions, particularly given that the two can be difficult to disentangle [25] and are likely most typically used in combination by clinicians [24]. Therefore, where exposure therapy has featured as a core component of a CBT intervention, significant research exists to support the efficacy [12, $22,26,27]$ and effectiveness $[12,28]$ of disorder specific exposure therapy techniques for the anxiety disorders, particularly interoceptive exposure [12, 22, 26-28]; OCD, particularly ERP $[12,22,29]$; and PTSD, particularly imaginal exposure [12, 22, 30]. Treatment outcomes using a CBT approach have also proven to be long-lasting, with patients maintaining symptom improvement for many years post-treatment [31]. Within the CBT package, research demonstrates that exposure therapy is a core component of this intervention, accounting for a significant proportion of treatment effects [12, 26, 27, 32 ]. When compared with pharmacotherapy interventions, exposure based treatments also deliver greater benefits long term [33] and are preferred by both adult clients and the caregivers of child clients [34, 35]. As a consequence, exposure based interventions are typically considered internationally as a first line intervention for the anxiety and related disorders [17] and features as a core component in manualized treatment programs.

Despite the substantial research supporting the efficacy and effectiveness of exposure therapy, research on its use in clinical practice has produced mixed results. Much of this research has come from the US [18, 36-42]. One large US based study of licensed doctoral level psychologists reported that $26 \%$ of therapists rarely or never used exposure therapy for obsessive compulsive disorder (OCD), 76\% rarely or never used interoceptive exposure for panic disorder, and 53\% rarely or never used therapist directed in-vivo exposure [37].

This pattern of use has been observed throughout Europe and the United Kingdom [43-45] and has been replicated across disorders; including Post Traumatic Stress Disorder (PTSD) [36, 45, 46], panic disorder [37, $47,48]$, OCD [18, 44, 49], and social phobia $[18,50]$ and age groups; including children and youth $[42,51]$; and adults [23]. Importantly, much of this research has been undertaken utilizing heterogeneous samples including psychologists, social workers and counselors $[18,38,45$, $51,52]$.
With homogenous samples of psychologists with specialist training in CBT however, the use of exposure therapy is considerably higher. In the US, 65\% reported use of interceptive exposure for panic disorder [53], 88.4\% used in-session exposure for social anxiety disorder [54] and $95 \%$ reported the use of exposure response prevention (ERP) for OCD [55]. Similarly, findings from the Netherlands suggest that $97.8 \%$ of clinicians reported use of exposure therapy for the anxiety and related disorders [56]. However, disorder specific exposure interventions were used with less frequency [56]. In particular, this research found that approximately $22 \%$ of clients with panic disorder do not receive interoceptive exposure. One interesting finding from this research is that exposure therapy was found to be most frequently used outside of sessions [56].

Taken together, there is mixed evidence for the use of exposure therapy in clinical practice, particularly evidence to suggest that use of disorder specific exposure interventions may warrant particular attention. This is consistent with literature which argues that dissemination and implementation efforts must be considered a key priority for our profession [57]. However, these findings are mostly based on samples of clinicians from the US and Europe, and it is unknown if the same is evident in Australia, and particularly amongst Australian psychologists. Formal training of psychologists varies across countries. In Australia, CBT, including exposure therapy, is the primary therapeutic training and the most recommended treatment approach for anxiety conditions. It is therefore important to understand the pattern of use within the Australian context.

This study sought to examine the use of exposure therapy in a homogenous sample of psychologists working in clinical practice in Australia who treat clients with an anxiety disorder, OCD or PTSD. The use of a homogenous psychologist sample is particularly important to the Australian context where psychologists are the primary group providing psychological intervention to individuals with mental health problems. Consistent with prior research, it was hypothesized that: (1) use of general exposure therapy techniques will be high amongst practicing psychologists; and (2) the following variables will be positively related to frequency of use: registration status, experience, training, and cognitive-behavioural orientation.

\section{Method}

\section{Participants}

A total of 164 participants commenced this study between December 2018 and December 2019. Participants who met exclusion criteria and those with incomplete data were not included in final analyses. Figure 1 


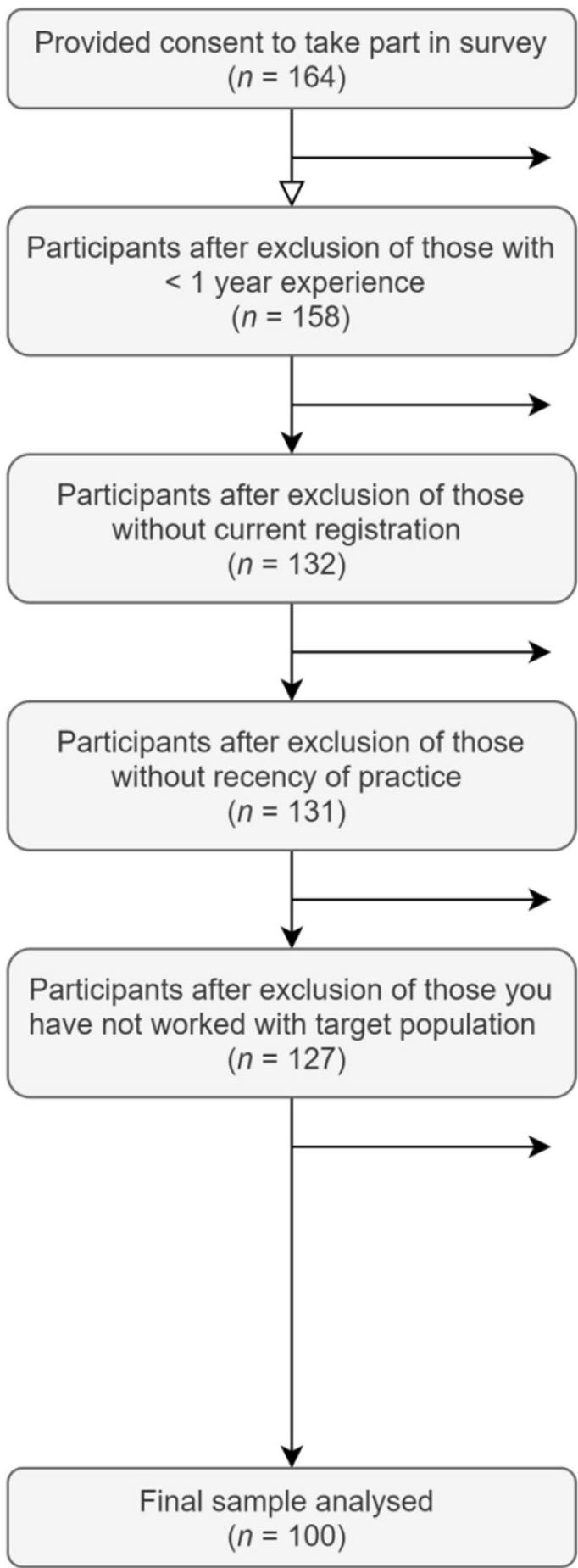

Exclusion: $<1$ year clinical experience $(n=26)$

$$
(n=1)
$$

\section{Exclusion: nil clinical work in last 12 months $(n=1)$}

\section{Exclusion: nil clinical work with target population in last 12 months

$$
(n=19)
$$

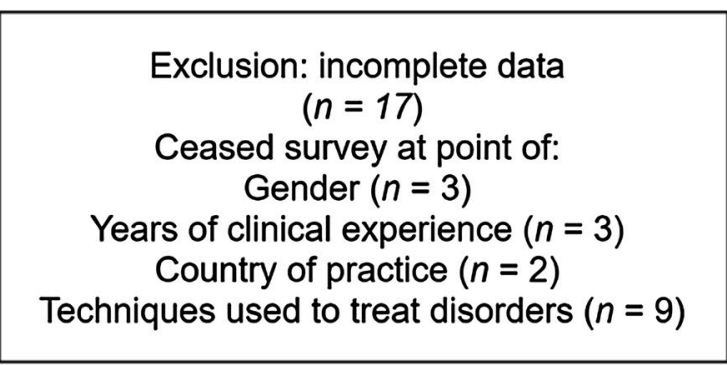

Fig. 1 Flowchart depicting participant inclusion and exclusion

outlines details of participant inclusion and exclusion. The final sample included 100 registered psychologists $\left(M_{\text {age }}=40.60 ; \mathrm{SD}=10.78\right.$; range 23 to 71 years; $84 \%$ female). As recruitment source and completion rates were not monitored in this study, response rate is unavailable. This sample was also utilized to examine barriers to the use of exposure therapy in a study reported elsewhere. To be included in the study participants were required to: (1) be a registered or provisionally registered psychologists, (2) have more than 1 year of experience 
in the practice of psychology, (3) have worked clinically, to any frequency, in the last 12 months, and (4) regularly see clients with a diagnosis of or symptoms consistent with an anxiety disorder, OCD and / or PTSD. Table 1 outlies the demographic information of the sample. The study received ethical approval from the University Human Research and Ethics Committee (Approval code: H12488).

\section{Measures}

\section{Demographic questionnaire}

The demographic questionnaire asked participants to indicate their (1) gender; (2) age; (3) years of clinical experience; (4) current registration status; (5) whether they have worked clinically in the last 12 months; (6) the country they currently practise in; (7) highest level of completed training; (8) how frequently they see clients with anxiety and related disorders; and (9) primary theoretical orientation. The demographic questionnaire can be found in the Additional file 1.

\section{Exposure therapy use questionnaire}

The exposure therapy use questionnaire was adapted from Hipol and Deacon [18] and Freiheit et al. [37]. Use was assessed by asking questions related to the frequency of exposure use and frequency of use of other therapeutic

Table 1 Participant Demographics for Total Sample $(N=100)$

\begin{tabular}{ll}
\hline Variable & $\mathbf{N}$ \\
\hline Gender (\% female) & 84 \\
Years of clinical experience & \\
$1-5$ years & 34 \\
$6-10$ years & 18 \\
$11-15$ years & 25 \\
15+years & 23 \\
Registration status & \\
Fully registered with endorsement & 60 \\
Fully registered (generalist psychologists) & 27 \\
Provisionally registered (in training) & 13 \\
Highest level of training & \\
Research PhD & 19 \\
Clinical masters/doctorate & 65 \\
Master of psychology & 8 \\
Bachelor's degree & 8 \\
Theoretical orientation & \\
Cognitive behavior therapy & 75 \\
Eclectic & 10 \\
Acceptance and commitment therapy & 9 \\
Psychodynamic & 23 \\
Schema focused therapy & \\
Client centered & 3 \\
\hline
\end{tabular}

techniques when working with clients with an anxiety disorder, OCD and/or PTSD. Response options were requested on a 5-point Likert scale and included 'never', 'sometimes', 'half the time', 'often', and 'very often'. It then asked questions about treatments considered most essential to treatment effectiveness, in order of priority when working with clients with anxiety, OCD and PTSD, highlighting the top three techniques. The exposure therapy use questionnaire can be found in the Additional file 1.

\section{Procedure}

Participants were identified and invited to participate in the online study via university psychology training programs, advertisements on relevant social media websites/ public list serves, and direct approach to psychologists via email. Recruitment source was not monitored in this study and participants were invited to enter a prize draw to win one of four $\$ 100$ gift vouchers for participation. Potential participants were directed to a link to the online questionnaire which opened to the participation information sheet and consent form, followed by the exposure therapy use and optimal use questionnaire. Importantly, only those who indicated that they work with each presentation were asked questions relating to frequency of intervention use. The questions appeared in fixed order and took approximately $15 \mathrm{~min}$ to complete.

\section{Data analysis}

The use of therapy techniques was investigated through descriptive statistics, one-way repeated MANOVA univariate analysis and planned comparisons to determine differences in exposure therapy use by registration, training and years of experience. To understand the use of techniques considered essential to treatment, Chi square analyses were undertaken. Group differences in use were analyzed via multiple linear regression, independent sample $t$ test and Pearson product-moment correlations were performed to determine if frequency of working with the population was predictive of exposure use.

Effect sizes were calculated and interpreted as suggested by Cohen [58]. Effect sizes for analysis of variance (Cohen's $f$ ) were interpreted as small $=.10$, medium $=.25$ and large $=.40$; effect sizes for chi-square (Cohens $\mathrm{w}$ ) were interpreted as small $=.10$, medium,.$=30$ and large $=.50$; effect sizes for regression (Cohen's $f^{2}$ ) were interpreted as small $=.02$, medium $=.15$, and large $=.35$; effect sizes of independent means (Cohen's $d$ ) were interpreted as small $=.20$, medium $=.50$, and large $=.80$, and effect sizes for correlations were (Cohen's $r$ ) were interpreted as small $=.10$, medium $=.30$ and large $=.50$ [58].

An a priori power analysis indicated that with a medium to large effect size, alpha of .05 and power of .95, 
108 participants were required to assess observed and expected data; 87 participants were required to assess group differences in use by registration, training and experience; and 111 participants were required to determine if frequency of work with population is predictive of use. All analyses were conducted using IBM SPSS Statistical Software Version 25.

\section{Results}

\section{Use of exposure therapy for anxiety disorders, OCD and PTSD}

Participants were asked to provide data on techniques used for presentations reported as frequently treated over the preceding 12 months. When assessing general use, $95.88 \%$ of participants reported general use of exposure therapy when working with clients with an anxiety disorder, $91.67 \%$ reported use when working with clients with OCD, and $82.35 \%$ reported general use when working with clients with PTSD. Table 2 presents the frequency with which participants reported often or very often use of specific therapeutic techniques when working with each disorder. The three most frequently reported techniques used when working with clients with (a) an anxiety disorder, included cognitive restructuring, elimination of avoidance and safety behaviours, and self-directed in-vivo exposure; (b) OCD, included ERP, elimination of avoidance and safety behaviours and selfdirected in-vivo exposure and (c) PTSD, included cognitive restructuring, elimination of avoidance and safety behaviours, and equal use was reported for imaginal exposure and breathing retraining.

Results of one-way repeated MANOVA revealed a statistically significant difference in use across disorders for the elimination of avoidances and safety behaviours $F(2,68)=6.00, p<.004$; Wilk's $\Lambda=.85$; imaginal exposure $F(2,68)=15.34, p<.00$; Wilk's $\Lambda=.69$; motivational interviewing; $F(2,68)=4.39, p<.02$; Wilk's $\Lambda=.89$; EMDR $F(2,68)=6.79, p<.002$; Wilk's $\Lambda=.83$; and ERP $F(2,68)=62.41, p<.00$; Wilk's $\Lambda=.35$. Univariate analysis and Tukey post hoc test revealed that use of elimination of avoidance and safety behaviours was statistically higher with the anxiety disorders $(M=.58, S D=.49)$ than $\operatorname{OCD}(M=.39, S D=.49)$ and PTSD $(M=.37, S D=.49)$; use of imaginal exposure was statistically higher for

Table 2 Use of treatment techniques by Australian psychologists for the anxiety disorders, OCD and PTSD (often and very often use), results of the 5 -point Likert scale and (M, SD) and differences in use across techniques

\begin{tabular}{|c|c|c|c|c|c|c|c|c|}
\hline Intervention & $\begin{array}{l}\text { Anxiety } \\
(\%) \\
(n=97)\end{array}$ & $\begin{array}{l}\text { OCD (\%) } \\
(n=72)\end{array}$ & $\begin{array}{l}\text { PTSD } \\
(\%) \\
(n=68)\end{array}$ & Anxiety $M(\mathrm{SD})$ & $\mathrm{OCD} M(\mathrm{SD})$ & PTSD $M($ SD) & $\begin{array}{l}\text { Wilk's } \\
\Lambda\end{array}$ & $p$ value \\
\hline \multicolumn{9}{|l|}{ Exposure } \\
\hline Self-directed in-vivo exposure & 58.8 & 65.3 & 44.1 & $3.48(1.31)$ & $3.78(1.42)$ & $3.00(1.51)$ & .97 & .37 \\
\hline Therapist assisted in-vivo exposure & 42.3 & 55.6 & 27.9 & $2.87(1.41)$ & $3.46(1.49)$ & $2.54(1.43)$ & .99 & .83 \\
\hline ERP & 40.2 & 83.3 & 19.1 & $3.02(1.38)$ & $4.36(1.14)$ & $2.15(1.37)$ & .35 & .00 \\
\hline Imaginal exposure & 36.1 & 48.6 & 47.1 & $2.83(1.32)$ & $3.13(1.40)$ & $3.29(1.46)$ & .69 & .00 \\
\hline Interoceptive exposure & 26.8 & 18.1 & 19.1 & $2.37(1.36)$ & $2.18(1.23)$ & $2.13(1.29)$ & 1.00 & 1.00 \\
\hline \multicolumn{9}{|l|}{ CBT (non-exposure) } \\
\hline Cognitive restructuring & 72.2 & 50 & 60.3 & $3.93(1.14)$ & $3.33(1.31)$ & $3.63(1.19)$ & .93 & .07 \\
\hline Elimination of avoidance and safety behaviours & 69.1 & 76.4 & 57.4 & $\begin{array}{l}3.99 \\
(1.08)\end{array}$ & $4.08(1.33)$ & $3.38(1.37)$ & .85 & .00 \\
\hline Breathing retraining & 45.4 & 31.9 & 47.1 & $\begin{array}{l}3 / 09 \\
(1.41)\end{array}$ & $2.56(1.55)$ & $3.13(1.54)$ & .94 & .14 \\
\hline Progressive muscle relaxation & 33 & 16.7 & 38.2 & $2.68(1.21)$ & ${ }^{2}(1.29)$ & $2.71(1.40)$ & .98 & .50 \\
\hline \multicolumn{9}{|l|}{ Other } \\
\hline Mindfulness & 41.2 & 23.6 & 41.2 & $3.01(1.36)$ & $2.43(1.32)$ & $3.03(1.32)$ & .93 & .08 \\
\hline Motivational interviewing & 24.7 & 25 & 16.2 & $2.58(1.24)$ & $2.38(1.36)$ & $2.15(1.21)$ & .89 & .02 \\
\hline Acceptance and commitment therapy & 21.6 & 22.2 & 16.2 & $2.31(1.29)$ & $2.18(1.33)$ & $2.09(1.22)$ & .99 & .82 \\
\hline Other & 19.6 & 18.1 & 20.6 & $1.83(1.49)$ & $1.73(1.41)$ & $1.81(1.47)$ & .96 & .26 \\
\hline Meditation & 14.4 & 11.1 & 10.3 & $2.04(1.17)$ & $1.73(1.11)$ & $1.88(1.15)$ & .99 & .32 \\
\hline Dialectical behavior therapy & 10.3 & 0 & 14.7 & $1.86(1.09)$ & $1.39(0.66)$ & $1.96(1.25)$ & .91 & .05 \\
\hline Psychodynamic psychotherapy & 8.2 & 4.2 & 10.3 & $1.55(1.04)$ & $1.26(0.75)$ & $1.51(1.07)$ & .93 & .08 \\
\hline EMDR & 6.2 & 4.2 & 14.7 & $1.38(1.41)$ & $1.25(0.76)$ & $1.69(1.28)$ & .83 & .00 \\
\hline Hypnosis & 1.0 & 4.2 & 1.5 & $1.07(0.36)$ & $1.17(0.69)$ & $1.15(1.37)$ & .99 & .32 \\
\hline
\end{tabular}


PTSD $(M=.43, S D=.49)$ than with anxiety $(M=.05$, $S D=.22)$ and $\mathrm{OCD}(M=.12, S D=.33)$; use EMDR was statistically higher for PTSD $(M=.20, S D=.40)$ than with anxiety $(M=.02, S D=.14)$ and $\mathrm{OCD}(M=.01, S D=.12)$; and use of ERP was statistically different across all disorders, greatest use with $\mathrm{OCD}(M=.77, S D=.42)$, followed by anxiety $(M=.34, S D=.48)$, and PTSD $(M=.07$, $S D=.26)$. No statistically significant differences were found in use of motivational interviewing across disorders. See Table 2 for results.

Techniques reported as most essential to the effectiveness of treatment for the anxiety disorders, OCD and PTSD

Participants were asked to indicate, in order of priority, the three techniques they see as most essential to treatment effectiveness. Results are presented in Table 3. Descriptive statistics suggest that the three techniques reported as being most essential to the treatment effectiveness of the anxiety disorders included the use of cognitive therapy (66\%), elimination of avoidance of safety behaviours (57.7\%), and equally self-directed invivo exposure (34\%)/ERP (34\%). The three techniques reported as being most essential to the treatment effectiveness of OCD included ERP (80.6\%), cognitive restructuring (51.4\%), and elimination of avoidance and safety behaviours $(40.3 \%)$. The three techniques reported as being most essential to the treatment effectiveness of PTSD included the use of cognitive restructuring (66.2\%), imaginal exposure (44.1\%), and elimination of avoidance and safety behaviours (38.2\%).

When considering the essential use of specific techniques for each disorder, Chi-square analysis revealed a significant relationship between disorder and essentiality of ERP $X^{2}(2)=76.69, p=.00$, representing a large effect, where ERP was considered most essential to the treatment of OCD (58, 77.3\%). Imaginal exposure $X^{2}(2)=41.64, p=.00$ (large effect) was considered most essential to the treatment of PTSD $(30,42.9 \%)$. Elimination of avoidance and safety behaviours $X^{2}(2)=9.21$, $p=.01$, (small effect) was considered most essential to the treatment of anxiety $(56,57.7 \%)$ and EMDR $X^{2}(2)=25.42, \quad p=.00$, (medium effect) was considered most essential to the treatment of PTSD (14, 20\%). Results are presented in Table 3.

\section{Group differences in the use of exposure therapy (general) Anxiety disorders}

A multiple linear regression was undertaken to predict use of general exposure techniques for anxiety by registration, training, and years of experience. These variables statistically significantly predicted use $F(3,93)=8.14, p$ $.00, R^{2}=.21$, representing a medium effect. Registration

Table 3 Essential techniques for the treatment of the anxiety disorders, OCD and PTSD

\begin{tabular}{|c|c|c|c|c|c|}
\hline Intervention & $\begin{array}{l}\text { Anxiety (\%) } \\
(n=97)\end{array}$ & OCD (\%) $(n=72)$ & $\begin{array}{l}\text { PTSD (\%) } \\
(n=68)\end{array}$ & $X^{2}$ & $p$ value \\
\hline \multicolumn{6}{|l|}{ Exposure } \\
\hline ERP & 34.00 & 80.60 & 7.40 & 76.79 & .00 \\
\hline Self-directed in-vivo exposure & 34.00 & 38.90 & 23.50 & 3.06 & .22 \\
\hline Therapist assisted in-vivo exposure & 22.70 & 15.30 & 22.10 & .19 & .91 \\
\hline Imaginal exposure & 5.20 & 12.50 & 44.10 & 41.64 & .00 \\
\hline Interoceptive exposure & 3.10 & 4.20 & 4.40 & .19 & .91 \\
\hline \multicolumn{6}{|l|}{ CBT (non-exposure) } \\
\hline Cognitive restructuring & 66.00 & 51.40 & 66.20 & 5.94 & .05 \\
\hline Elimination of avoidance and safety behaviours & 57.70 & 40.30 & 38.20 & 9.21 & .01 \\
\hline Breathing retraining & 22.70 & 9.70 & 19.10 & 5.35 & .07 \\
\hline Progressive muscle relaxation & 7.20 & 2.80 & 4.40 & 1.95 & .38 \\
\hline \multicolumn{6}{|l|}{ Other } \\
\hline Acceptance and commitment therapy & 11.30 & 5.60 & 8.80 & 1.93 & .38 \\
\hline Mindfulness & 10.30 & 5.60 & 17.60 & 5.30 & .07 \\
\hline Other & 6.20 & 4.20 & 8.80 & 1.30 & .52 \\
\hline Motivational interviewing & 5.20 & 5.60 & 0 & 3.81 & .15 \\
\hline Psychodynamic psychotherapy & 5.20 & 0 & 4.40 & 3.81 & .15 \\
\hline Dialectical behavior therapy & 3.10 & 0 & 5.90 & 4.23 & .12 \\
\hline EMDR & 2.10 & 1.40 & 20.60 & 25.42 & .00 \\
\hline Meditation & 1.00 & 0 & 0 & 1.50 & 4.7 \\
\hline Hypnosis & 0 & 1.40 & 0 & 2.23 & .33 \\
\hline
\end{tabular}


and training added statistically significantly to the prediction.

An independent $t$ test was conducted to examine whether group differences in the use of exposure therapy were observed between clinicians who reported a CBT versus all other orientations. No statistically significant differences between CBT and other reported orientations was found $t(95)=1.11, p=.27$.

A Pearson product-moment correlation was run to determine the relationship between time spent working with clients with anxiety, OCD and PTSD and the general use of exposure therapy with clients with an anxiety disorder. There was a small, positive correlation between time spent working with the population and use, which was statistically significant $(r=.24, n=97, p=.02)$.

\section{$O C D$}

A multiple linear regression was undertaken to predict use of general exposure techniques for OCD by registration, training, and years of experience. These variables statistically significantly predicted use $F(3,68)=3.41, p$ $.02, R^{2}=.13$, representing a medium effect.

An independent sample $t$ test was conducted to examine whether group differences in the use of exposure therapy was observed between clinicians who reported a CBT versus all other orientations. No statistically significant difference for the use of ERP between participants who identified their theoretical orientation as being CBT versus other theoretical orientations, $t(70)=.32, p=.21$ was found.

A Pearson product-moment correlation was run to determine the relationship between time spent working with clients with anxiety, OCD and PTSD and use of general exposure techniques with clients with OCD. No statistically significant correlation was found $(r=.08, n=72$, $p=.53)$.

\section{PTSD}

A multiple linear regression was undertaken to predict use of general exposure techniques for PTSD by registration, training, and years of experience. These variables statistically significantly predicted use $F(3,64)=3.87, p$ $.01, R^{2}=.15$, representing a medium effect. Level of training added statistically significantly to the prediction.

An independent sample $t$ test was conducted to examine whether group differences in the use of exposure therapy were observed between clinicians who reported a CBT versus all other orientation for the treatment of PTSD. Results indicated no statistically significant differences between use $t(66)=.09, p=.96$.

A Pearson product-moment correlation was run to determine the relationship between time spent working with clients with anxiety, OCD and PTSD and use of exposure therapy with clients with PTSD. A small positive correlation was found $(r=.27, n=68, p=.03)$.

\section{Group differences in the use of exposure therapy (disorder specific) \\ Anxiety}

A multiple linear regression was undertaken to predict use of imaginal exposure for anxiety by registration, training, and years of experience. These variables statistically significantly predicted use $F(3,93)=4.28, p .01$, $R^{2}=.12$, representing a small effect. Registration added statistically significantly to the prediction.

A multiple linear regression was undertaken to predict use of interoceptive exposure for anxiety by registration, training, and years of experience. These variables statistically significantly predicted use $F(3,93)=7.47, p$ $.00, R^{2}=.19$, representing a medium effect. Registration added statistically significantly to the prediction.

An independent sample $t$ test was conducted to examine whether group differences in the use of interoceptive exposure was observed between clinicians who reported a CBT versus all other orientations. No statistically significant difference for use between participants who identified their theoretical orientation as being CBT versus all other theoretical orientations, $t(95)=.16, p=.88$ was found.

A Pearson product-moment correlation was run to determine the relationship between time spent working with clients with anxiety, OCD and PTSD and use of interoceptive exposure with clients with anxiety. A medium positive correlation was found $(r=.42, n=97$, $p=.00$ ). Similarly, a medium positive correlation was found for the use of imaginal exposure with clients with anxiety $(r=.42, n=97, p=.00)$.

\section{PTSD}

A multiple linear regression was undertaken to predict use of imaginal exposure for PTSD by registration, training, and years of experience. These variables statistically significantly predicted use $F(3,64)=5.84, p .00, R^{2}=.22$, representing a medium effect. Registration added statistically significantly to the prediction, $p=.01$.

An independent sample $t$ test was conducted to examine whether group differences in the use of imaginal exposure was observed between clinicians who reported a CBT versus other orientation. No statistically significant difference for use between participants who identified their theoretical orientation as being CBT versus other theoretical orientations, $t(51)=.36, p=.72$ was found.

A Pearson product-moment correlation was run to determine the relationship between time spent working 
with clients with anxiety, OCD and PTSD and use of imaginal exposure with clients with PTSD. No statistically significant correlation was found $(r=.01, n=68$, $p=.95)$.

\section{$O C D$}

A multiple linear regression was undertaken to predict use of ERP for OCD by registration, training, and years of experience. These variables statistically significantly predicted use $F(3,68)=7.11, p .00, R^{2}=.24$, representing medium effect. Level of training added statistically significantly to the prediction, $p=.00$.

An independent sample $t$ test was conducted to examine whether group differences in the use of ERP was observed between clinicians who reported a CBT versus other orientation. No statistically significant difference for the use of ERP between participants who identified their theoretical orientation as being CBT versus other theoretical orientations, $t(70)=.59, p=.55$ was found.

A Pearson product-moment correlation was run to determine the relationship between time spent working with clients with anxiety, OCD and PTSD and use of ERP with clients with OCD. No statistically significant correlation was found ( $r=0.14, n=72, p=0.24)$.

\section{Discussion}

The aim of the present study was to examine the use of exposure therapy in a homogenous sample of psychologists working in clinical practice in Australia who treat clients with an anxiety disorder, OCD and/or PTSD. It was hypothesized that: (1) general use of exposure therapy will be high amongst clinicians; and that, (2) registration status, experience, training and cognitivebehavioural orientation would be positively related to frequency of use.

In this study, participants' general use of exposure therapy was high with $95 \%$ reporting use with anxiety, $91 \%$ reporting use with OCD and $82 \%$ reporting use with PTSD. While the general use of exposure therapy was high, the frequency of use of disorder specific exposure techniques varied. Of note, the use of interceptive exposure for anxiety symptoms was reported by only $26 \%$ of participants, while the frequent use of imaginal exposure for PTSD was reported by $47 \%$ of participants. Importantly, this was not the case in relation to the treatment of OCD, where ERP was reported to be frequently used by $83 \%$ of participants. These findings are similar to research from the Netherlands [56] and US [53, 54], which reported high general use amongst homogenous samples of psychologists. They however differ from studies based on heterogenous samples of general mental health workers, where use was consistently found to be lower $[18,38,45,52]$. The differences are likely due to the use of a homogenous sample in the current study, and that the training of psychologists in Australia has a strong CBT focus, resulting in a higher use of this treatment technique.

When specifically considering use compared across disorders, imaginal exposure was found to be used more frequently for PTSD, and ERP was used most frequently for OCD. This is a pleasing finding, suggesting that there is increasing awareness of the importance of using disorder specific interventions with particular disorders. Further to this, consistent with existing research on treatment effectiveness [12, 26, 29], the current study's results suggest that ERP was considered by the sample to be essential to the treatment of OCD and imaginal exposure was considered essential to the treatment of PTSD. No other significant relationships were found between use of general or specific exposure techniques for anxiety, OCD or PTSD. Given that these exposure techniques are considered first line interventions for the treatment of anxiety, OCD and PTSD [15-17], this finding is concerning and warrants further attention in dissemination and implementation efforts, considered to be a priority for psychology [57].

Across disorders, registration and training were most frequently found to be positively associated with the use of exposure therapy, although importantly, this association was not consistenly observed across disorders. This finding is consistent with prior research which suggests that training is positively correlated with use [56]. This finding may be explained by the fact that higher levels of training may be associated with greater attention to specialised areas of practice training, including the use of disorder specific interventions. Time spent working with these disorders was found to be associated with exposure use across the anxiety disorders, both general and disosrder specific interventions, but not for OCD or PTSD presentations. This finding is consistent with recent research undertaken in the US [53-55], suggesting greater use of evidence based practice with specalisation.

Years of experience and theoretical orientation were not found to be associated with the use of general or specific exposure therapy use across disorders. The former may be explained by therapist drift [59], whilst the latter may be due to the fact that the majority of this sample reported their primary theoretical orientation as CBT, which is also, currently and historically, the predominant technique taught in university education settings $[15,19]$.

Whilst this study did not seek to assess the quality of exposure techniques utilised, results do suggest that other techniques, particularly cognitive restructuring, are frequently used and typically considered essential components to treatment effectiveness. Results may suggest that these techniques are used in combination with exposure 
techniques, suggesting modified use of exposure therapy. This is again a similar finding to that reported in previous research $[18,56]$ where both homogenous and heterogenous samples have been used. This is thought to represent a concerning modified pattern of exposure therapy use in clinical practice [18].

To the authors' knowledge, this study is the first of its kind to assess the use of exposure therapy in an Australian sample of psychologists. Results are promising, suggesting that many clinicians in Australia utilise general exposure techniques, both within and between sessions with the exception of PTSD. However, work remains necessary to improve the use of disorder specific interventions. In particular, the low percentage use of interoceptive exposure with anxiety, and the low percentage use of imaginal exposure with PTSD needs to be better understood. To address this, we recommend that future research considers the barriers to the use of these techniques, and how to address these within training and professional development programs. To be most effective, training programs should be evaluated to ensure effectiveness of dissemination and implementation, which is currently not routinely undertaken.

Notwithstanding the important findings reported in this study, there are some notable limitations. Firstly, this study has utilized a relatively small and female dominated sample, although importantly is representative of psychologists in Australia on both gender and registration status [60]. Similarly, a predominantly CBT orientation may have further impacted results obtained. Secondly, the study utilized self-report data, which included the use of exposure therapy in the project title. Results therefore may be impacted by reporting and selection bias [61]. Thirdly, participants were not asked to report on use of exposure therapy for each specific anxiety disorder, but rather across the anxiety disorders. Given the exposure therapy use may appropriately vary across anxiety disorders (e.g. GAD), this may have impacted on responses provided and results obtained. Fourthly, this study focused on the type and frequency of use of exposure techniques and did not assess the quality of use of exposure techniques in clinical practice, which is an important consideration for future research. Finally, whilst data was collected prior to the COVID-19 global pandemic, such events are likely to have a significant impact on the use of exposure therapy in clinical practice. Future research may wish to further explore this impact, both in clinician use and realistic client engagement.

In conclusion, the findings in this study suggest that the general use of exposure therapy by psychologists is high. However, the use of disorder specific exposure therapy techniques varies considerably amongst practicing psychologists, with the exception of ERP for OCD. Understanding when and how those techniques are used may inform knowledge and practice gaps, and formal and ongoing professional training needs. In particular, further training may be required in disorder specific exposure therapy interventions, to build on the fundamentals of the general exposure techniques and optimize disorder specific therapeutic outcomes. It is hoped that this research will contribute to the very important dissemination and implementation efforts currently being discussed in the broader psychological community.

\section{Supplementary Information}

The online version contains supplementary material available at https://doi. org/10.1186/s40359-021-00613-7.

Additional file 1. Exposure therapy use questionnaire.

\section{Acknowledgements}

Not applicable.

\section{Authors' contributions}

KM contributed to the conception, design, analysis and interpretation of the data. KM wrote the manuscript. TM and CG contributed to the conception, design, analysis and interpretation of the data. All authors read, revised and approved the final manuscript.

\section{Funding}

This research was funded by the School of Psychology, Western Sydney University.

\section{Availability of data and materials}

The datasets generated and analysed during the current student are not publicly available as this is subject to ongoing use for the purposes of PhD completion, but are available from the author on reasonable request.

\section{Declarations}

\section{Ethics approval and consent to participate}

This research was performed in accordance with the Declaration of Helsinki and was approved by the Western Sydney University Human Research and Ethics Committee (Approval code: H12488). All participants provide informed consent to participate in this research prior to any participation.

\section{Consent for publication}

Written informed consent for publication was obtained from all participants.

\section{Competing interests}

The authors declare that they have no competing interests.

\section{Author details}

${ }^{1}$ School of Psychology, Western Sydney University, Locked Bag 1797, Penrith, NSW 2751, Australia. ${ }^{2}$ Translational Health Research Institute, Western Sydney University, Locked Bag 1797, Penrith, NSW 2751, Australia.

Received: 6 January 2021 Accepted: 28 June 2021

Published online: 27 July 2021

\section{References}

1. Lawrence D, Hafekost J, Johnson SE, Saw S, Buckingham WJ, Sawyer $M G$, et al. Key findings from the second Australian child and adolescent survey of mental health and Wellbeing. Aust N Z J Psychiatry. 2016;50(9):876-86. 
2. Henderson S, Andrews G, Hall W. Australia's mental health: an overview of the general population survey. Aust N Z J Psychiatry. 2000;34(2):197-205.

3. Beesdo K, Knappe S, Pine DS. Anxiety and anxiety disorders in children and adolescents: developmental issues and implications for DSM-V. Psychiatr Clin. 2009;32(3):483-524

4. Destoop M, Van Den Eede F, Ansseau M, Albert A, Vanbelle S, Mignon A, et al. Prevalence and clinical characteristics of remission during treatment in generalized anxiety. Int J Psychiatry Clin Pract. 2013;17(2):90-7.

5. Fineberg NA, Hengartner MP, Bergbaum C, Gale T, Rössler W, Angst J. Lifetime comorbidity of obsessive-compulsive disorder and subthreshold obsessive-compulsive symptomatology in the community: impact, prevalence, socio-demographic and clinical characteristics. Int J Psychiatry Clin Pract. 2013;17(3):188-96.

6. Ginsburg GS, Kendall PC, Sakolsky D, Compton SN, Piacentini J, Albano $A M$, et al. Remission after acute treatment in children and adolescents with anxiety disorders: findings from the CAMS. J Consult Clin Psychol. 2011;79(6):806.

7. Barlow DH. Anxiety and its disorders: the nature and treatment of anxiety and panic. New York: Guilford Press; 2004.

8. Kaufman J, Charney D. Comorbidity of mood and anxiety disorders. Depress Anxiety. 2000;12(S1):69-76.

9. Kendall PC, Compton SN, Walkup JT, Birmaher B, Albano AM, Sherrill J, et al. Clinical characteristics of anxiety disordered youth. J Anxiety Disord. 2010;24(3):360-5.

10. Mendlowicz MV, Stein MB. Quality of life in individuals with anxiety disorders. Am J Psychiatry. 2000;157(5):669-82.

11. Ohayon MM, Roth T. Place of chronic insomnia in the course of depressive and anxiety disorders. J Psychiatr Res. 2003;37(1):9-15.

12. Hunsley J, Elliott $K$, Therrien Z. The efficacy and effectiveness of psychological treatments for mood, anxiety, and related disorders. Can Psychol. 2014;55(3):161.

13. Olatunji BO, Cisler JM, Deacon BJ. Efficacy of cognitive behavioral therapy for anxiety disorders: a review of meta-analytic findings. Psychiat Clin. 2010;33(3):557-77.

14. Moses K, Gayed M, Chuah S, Wootton BM. The use of evidence-based assessment for anxiety disorders in an Australian sample. J Anxiety Disorders. 2020;102279.

15. Barrington J. Cognitive behaviour therapy: standards for training and clinical practice. Behaviour Change. 2006;23(4):227-38.

16. Australian Psychological Society. Evidence-based psychological interventions in the treatment of mental disorders: A review of the literature. (4th ed). 2018.

17. National Institute for Health and Care Excellence. Common mental health disorders: Identification and pathways to care. London: NICE; 2011.

18. Hipol LJ, Deacon B. Dissemination of evidence-based practices for anxiety disorders in Wyoming: a survey of practicing psychotherapists. Behav Modif. 2013;37(2):170-88.

19. Kazantzis N, Munro MJ. The emphasis on cognitive-behavioural therapy within clinical psychology training at Australian and New Zealand universities: a survey of program directors. Aust Psychol. 2011;46(1):49-54.

20. Weissman MM, Verdeli H, Gameroff MJ, Bledsoe SE, Betts K, Mufson L, et al. National survey of psychotherapy training in psychiatry, psychology, and social work. Arch Gen Psychiatry. 2006:63(8):925-34

21. American Psychiatric Association. Diagnostic and statistical manual of mental disorders (5th ed): Washington, DC. 2013.

22. Carpenter JK, Andrews LA, Witcraft SM, Powers MB, Smits JA, Hofmann SG. Cognitive behavioral therapy for anxiety and related disorders: a meta-analysis of randomized placebo-controlled trials. Depression Anxiety. 2018;35(6):502-14.

23. Deacon B, Farrell N, Kemp JJ, Dixon LJ, Sy JT, Zhang AR, et al. Assessing therapist reservations about exposure therapy for anxiety disorders: the Therapist Beliefs about Exposure Scale. J Anxiety Disord. 2013;27(8):772-80

24. Abramowitz JS, Deacon BJ, Whiteside SP. Exposure therapy for anxiety: Principles and practice: Guilford Publications; 2019.

25. McLean PD, Whittal ML, Thordarson DS, Taylor S, Söchting I, Koch WJ, et al. Cognitive versus behavior therapy in the group treatment of obsessive-compulsive disorder. J Consult Clin Psychol. 2001;69(2):205.

26. Olatunji BO, Cisler JM, Deacon BJ. Efficacy of cognitive behavioral therapy for anxiety disorders: a review of meta-analytic findings. Psychiatr Clin North Am. 2010;33(3):557-77.
27. Deacon B, Abramowitz JS. Cognitive and behavioral treatments for anxiety disorders: a review of meta-analytic findings. J Clin Psychol. 2004;60(4):429-41.

28. Stewart RE, Chambless DL. Cognitive-behavioral therapy for adult anxiety disorders in clinical practice: a meta-analysis of effectiveness studies. J Consult Clin Psychol. 2009;77(4):595.

29. Olatunji BO, Davis ML, Powers MB, Smits JA. Cognitive-behavioral therapy for obsessive-compulsive disorder: a meta-analysis of treatment outcome and moderators. J Psychiatr Res. 2013;47(1):33-41.

30. Watts BV, Schnurr PP, Mayo L, Young-Xu Y, Weeks WB, Friedman MJ. Meta-analysis of the efficacy of treatments for posttraumatic stress disorder. J Clin Psychiatry. 2013;74(6):541-50.

31. Wootton BM, Bragdon LB, Steinman SA, Tolin DF. Three-year outcomes of adults with anxiety and related disorders following cognitivebehavioral therapy in a non-research clinical setting. J Anxiety Disord. 2015:31:28-31.

32. Norton PJ, Price EC. A meta-analytic review of adult cognitive-behavioral treatment outcome across the anxiety disorders. J Nerv Ment Dis. 2007;195(6):521-31.

33. Barlow DH, Gorman JM, Shear MK, Woods SW. Cognitive-behavioral therapy, imipramine, or their combination for panic disorder: a randomized controlled trial. JAMA. 2000;283(19):2529-36.

34. Brown AM, Deacon BJ, Abramowitz JS, Dammann J, Whiteside SP. Parents' perceptions of pharmacological and cognitive-behavioral treatments for childhood anxiety disorders. Behav Res Ther. 2007;45(4):819-28.

35. Deacon B, Abramowitz JS. Patients' perceptions of pharmacological and cognitive-behavioral treatments for anxiety disorders. Behav Ther 2005;36(2):139-45.

36. Becker CB, Zayfert C, Anderson E. A survey of psychologists' attitudes towards and utilization of exposure therapy for PTSD. Behav Res Ther. 2004;42(3):277-92.

37. Freiheit SR, Vye C, Swan R, Cady M. Cognitive-behavioral therapy for anxiety: Is dissemination working? Behav Therapist. 2004; 27(2):25-32.

38. Cook JM, Biyanova T, Elhai J, Schnurr PP, Coyne JC. What do psychotherapists really do in practice? An Internet study of over 2,000 practitioners. Psychother Theory Res Pract Train. 2010;47(2):260.

39. Whiteside SPH, Sattler A, Ale CM, Young B, Hillson Jensen A, Gregg MS, et al. The use of exposure therapy for child anxiety disorders in a medical center. Prof Psychol Res Pract. 2016;47(3):206-14.

40. Mancebo MC, Eisen JL, Sibrava NJ, Dyck IR, Rasmussen SA. Patient utilization of cognitive-behavioral therapy for OCD. Behav Ther. 2011:42(3):399-412.

41. Goisman RM, Rogers MP, Steketee GS, Warshaw MG, Cuneo P, Keller M. Utilization of behavioral methods in a multicenter anxiety disorders study. J Clin Psychiatry. 1993;54(6):213-8.

42. Reid AM, Bolshakova MI, Guzick AG, Fernandez AG, Striley CW, Geffken $\mathrm{GR}$, et al. Common barriers to the dissemination of exposure therapy for youth with anxiety disorders. Commun Ment Health J. 2017;53(4):432-7.

43. Levita L, Duhne PGS, Girling C, Waller G. Facets of clinicians'anxiety and the delivery of cognitive behavioral therapy. Behav Res Ther. 2016;77:157-61.

44. Böhm K, Külz A. Versorgungsrealität der zwangsstörungen: werden expositionsverfahren eingesetzt? Verhaltenstherapie. 2008;18(1):18-24.

45. van Minnen A, Hendriks L, Olff M. When do trauma experts choose exposure therapy for PTSD patients? A controlled study of therapist and patient factors. Behav Res Ther. 2010;48(4):312-20.

46. Rosen CS, Chow HC, Finney JF, Greenbaum MA, Moos RH, Sheikh JI, et al. VA practice patterns and practice guidelines for treating posttraumatic stress disorder. J Traum Stress Off Publ Int Soc Traum Stress Stud. 2004;17(3):213-22.

47. Deacon BJ, Lickel JJ, Farrell NR, Kemp JJ, Hipol LJ. Therapist perceptions and delivery of interoceptive exposure for panic disorder. J Anxiety Disord. 2013;27(2):259-64.

48. Marcks BA, Weisberg RB, Keller MB. Psychiatric treatment received by primary care patients with panic disorder with and without agoraphobia. Psychiatr Serv. 2009;60(6):823-30.

49. Kozak MJ, Foa EB. Mastery of obsessive-compulsive disorder: a cognitivebehavioral approach. Oxford: Oxford University Press; 2005. 
50. Heimberg RG, Becker RE. Cognitive-behavioral group therapy for social phobia: basic mechanisms and clinical strategies. New York: Guilford Press; 2002.

51. Whiteside SPH, Deacon BJ, Benito K, Stewart E. Factors associated with practitioners' use of exposure therapy for childhood anxiety disorders. J Anxiety Disord. 2016;40:29-36

52. Reid AM, Guzick AG, Fernandez AG, Deacon B, McNamara JP, Geffken $G R$, et al. Exposure therapy for youth with anxiety: utilization rates and predictors of implementation in a sample of practicing clinicians from across the United States. J Anxiety Disord. 2018;58:8-17.

53. Wolf AW, Goldfried MR. Clinical experiences in using cognitive-behavior therapy to treat panic disorder. Behav Ther. 2014;45(1):36-46.

54. McAleavey AA, Castonguay LG, Goldfried MR. Clinical experiences in conducting cognitive-behavioral therapy for social phobia. Behav Ther. 2014;45(1):21-35.

55. Jacobson NC, Newman MG, Goldfried MR. Clinical feedback about empirically supported treatments for obsessive-compulsive disorder. Behav Ther. 2016:47(1):75-90.
56. Sars D, van Minnen A. On the use of exposure therapy in the treatment of anxiety disorders: a survey among cognitive behavioural therapists in the Netherlands. BMC Psychol. 2015;3(1):26.

57. McHugh RK, Barlow DH. The dissemination and implementation of evidence-based psychological treatments: a review of current efforts. Am Psychol. 2010;65(2):73.

58. Cohen J. A power primer. Psychol Bull. 1992;112(1):155.

59. Waller $\mathrm{G}$, Turner $\mathrm{H}$. Therapist drift redux: why well-meaning clinicians fail to deliver evidence-based therapy, and how to get back on track. Behav Res Ther. 2016;77:129-37.

60. Psychology Board of Australia: Registrant data 2019.

61. Althubaiti A. Information bias in health research: definition, pitfalls, and adjustment methods. J Multidiscip Healthc. 2016;9:211

\section{Publisher's Note}

Springer Nature remains neutral with regard to jurisdictional claims in published maps and institutional affiliations.
Ready to submit your research? Choose BMC and benefit from:

- fast, convenient online submission

- thorough peer review by experienced researchers in your field

- rapid publication on acceptance

- support for research data, including large and complex data types

- gold Open Access which fosters wider collaboration and increased citations

- maximum visibility for your research: over $100 \mathrm{M}$ website views per year

At $\mathrm{BMC}$, research is always in progress.

Learn more biomedcentral.com/submissions 